\title{
Preparation and characterization of copper oxide nanofluid for heat transfer applications
}

\author{
R. Manimaran $\cdot$ K. Palaniradja $\cdot$ N. Alagumurthi • \\ S. Sendhilnathan $\cdot$ J. Hussain
}

Received: 13 October 2012/ Accepted: 4 December 2012/Published online: 1 January 2013

(C) The Author(s) 2012. This article is published with open access at Springerlink.com

\begin{abstract}
Copper oxide nanofluid has been prepared by single-step wet chemical precipitation method. The preparation of nanofluid is carried out by reducing copper chloride with sodium hydroxide as reducing agent in deionised water as base fluid by means of conventional heating. The characterization of sample nano powder is done by X-ray diffraction (XRD), EDXA, SEM, and TGA. The mean size of the particle was determined from the XRD pattern by using the Scherrer formula and the average particle size was found to be $20 \mathrm{~nm}$. Also an increase in thermal conductivity of the prepared copper oxide nanofluid was found to be $12.4 \%$ compared to deionised water.
\end{abstract}

Keywords Copper oxide $\cdot$ XRD $\cdot$ Nanofluid $\cdot$ Thermal conductivity

\section{Introduction}

In many industrial applications, fluids are generally used as a cooling medium and the enhancement of the heat transfer behavior of these fluids is of great importance in many applications. Nanofluids are a new class of fluids engineered by Choi (1995) dispersing nanometer-sized materials (nanoparticles) in base fluids. In other words, nanofluids are nanoscale colloidal suspensions containing

R. Manimaran $(\bowtie) \cdot$ K. Palaniradja $\cdot$ N. Alagumurthi

J. Hussain

Department of Mechanical Engineering, Pondicherry

Engineering College, Pondicherry, India

e-mail: manimaranpec@gmail.com

S. Sendhilnathan

Department of Physics, Anna University of Technology,

Pattukotai Campus, Trichy, India condensed nanomaterials. They are two-phase systems with one phase (solid phase) in another (liquid phase). Nanofluids have been found to possess enhanced thermo physical properties such as thermal conductivity, thermal diffusivity, viscosity, and convective heat transfer coefficients compared to those of base fluids such as oil or water. It has demonstrated great potential applications in many fields such as microelectronics, transportation, manufacturing, heating, and cooling.

Lee et al. (1999) developed model for measuring the thermal conductivity of fluids containing metals or metal oxides of conductivity higher than base fluids. Wang et al. (1999) conducted experiments on the fluids having nanoparticles mixture and found that thermal conductivities of nanoparticle-fluid mixtures increase relative to those of the base fluids. Li and Peterson (2006) performed experiment on the nanofluids by varying the temperature of the fluid and also the volume fraction has been changed, and found that there is an effective increase in the thermal conductivity of the nanofluids. Xuan and Li (2003) studied the convective and flow features of the nanofluids. Liu et al. (2006) and Murshed et al. (2005) showed the thermal conductivity enhancement of $\mathrm{CuO}$ nanofluid and titanium dioxide nanofluid, respectively. Lazarus (Godson et al. 2010) found that the main reason for the heat transfer enhancement of nanofluids is that the suspended nanoparticles increase the thermal conductivity of the fluids, and the chaotic movement of ultrafine particles increases fluctuation and turbulence of the fluids, which accelerates the energy exchange process.

Copper oxide nanoparticles are an interesting class of material having multifunctional properties with promising applications in the areas of catalysts, batteries, magnetic storage media, solar energy, and superconductors (Frietsch et al. 2000; Sukhorukov et al. 1998; Carnes and Klabunde 2003). Several methods have been available to synthesis 
copper oxide nanoparticles with various morphologies. These nanoparticles have great advantages over conventional materials because of their large surface area. Formation of well-dispersed nanoparticles is a challenging issue because these particles tend to agglomerate with time and tend to lower the surface energy. Very high reaction rates are achieved when highly dispersed nanoparticles are used for hydro cracking, coal liquefaction, etc. Copper oxide is used as an ultra dispersed catalyst (Bennici et al. 2003; You et al. 2002) in heavy oil upgrading. Copper oxide nanoparticles are also used in nanofluids where dispersed nanoparticles are used for increasing the thermal conductivity of fluids.

Preparation of stable nanofluids is of very much important in the area of nanofluid research and its application. For the past decades, researches had used two-step methods for preparing nanofluids, for example, Eastman et al. (2001) used two-step method to prepare nanofluid and shown that copper nanometer-sized particles dispersed in ethylene glycol has a much higher effective thermal conductivity than pure ethylene glycol. Though this method is economic, the problem of drying, storage, and transportation exist. Also, the problem of agglomeration and clogging leads to reduced thermal conductivity of nanofluids.

As an example of one-step method, Jwo et al. (2007) prepared $\mathrm{CuO}$-water nanofluid using temperature arc method and indicated about the improvement of thermal conductivity of nanofluid by $9.6 \%$. Another example; Kurihara et al. (1995) used polyol as a solvent to prepare copper nanoparticles. Here, the drawback of this method is that the copper salt should be heated to its boiling point. Recently, Zhu et al. (2004) developed an efficient novel one-step chemical method for preparing copper nanofluids, and this method has the advantages in terms of controlling the particle size, reducing agglomeration of the nanoparticles and producing nanofluids in a large scale.

From the literature studies, it is very clear that copper oxide nanofluids has high thermal conductivity and also have good heat transfer properties suitable for thermal engineering applications. In this study, copper oxide nanofluids was synthesized with a wet chemical method, and the characterization has been carried out using XRD, EDXA, SEM, and TGA.

\section{Experimental section}

Copper oxide nanofluid has been prepared using wet chemical method. All the chemicals such as copper chloride, sodium hydroxide, and hydrochloric acid were purchased from Aldrich chemicals and they are in analytical grade. Since the method involves single-step process, the preparation of copper oxide nanoparticle is carried out along with nanofluids. In this preparation process, $100 \mathrm{ml}$ of deionised water is taken in a $500 \mathrm{ml}$ of beaker. To this 0.51 $\mathrm{g}$ of copper chloride was added along with one gram of sodium hydroxide pellets. The reaction mixture was heated along with magnetic stirring and the process is carried out for one hour. As a result, the color of the solution changes from blue to black after the reaction, and then the mixture was cooled to room temperature. Schematically the chemical reaction can be represented as

$\mathrm{CuCl}_{2}+2 \mathrm{NaOH}=\mathrm{CuO}+\mathrm{NaCl}+\mathrm{H}_{2} \mathrm{O}$

The PH value of the so formed copper oxide wet precipitate is neutralized by adding droplets of hydrochloric acid. Then the copper oxide wet precipitate is washed with deionised water to remove the impurity ions present in the solution. Copper oxide nanofluids are obtained by dispersing the wet precipitate into the required amount of deionised water under ultrasonic vibration for about $4 \mathrm{~h}$ to have uniform dispersion of copper oxide nanoparticles.

\section{Results and discussion}

\section{Characterization}

The particles were characterized by XRD for structural determination and estimation of crystalline size using the instrument PAnalytical X'Pert PRO diffractometer, Scanning Electron Microscope was done using the Hitachi, model:S-3400 N for surface morphology and XRF was carried out using the Bruker, model: S4 Pioneer for chemical composition. The thermal stability of the sample was investigated using the instrument: SDT Q600 V20.9 Build 20, and the thermal conductivity is measured using KD2 Pro thermal analyzer, Decagon Devices, Inc., USA.

\section{XRD}

The peaks apparent in the Fig. 1 indicate good crystallinity of the copper oxide nanoparticles, no characteristics peaks of any other phase of $\mathrm{CuO}$ were observed. X-ray diffraction studies confirmed that the synthesized materials were $\mathrm{CuO}$, and all the diffraction peaks agreed with that of monoclinic $\mathrm{CuO}$ according to the literature (JCPDS, File No. 01-0801916). The average crystal size is $20 \mathrm{~nm}$ which is calculated using Debye-Scherrer formula.

$D=\frac{0.89 \lambda}{\beta \cos \theta}$

where $\lambda$ is the $\mathrm{X}$-ray wavelength (1.5406), $\beta$ is the peak width, and theta is the Braggs angle. The Energy dispersive $\mathrm{X}$-ray analysis reveals the presence of $\mathrm{Cu}$ and $\mathrm{O}$ elements in the prepared copper oxide nanopowder, as shown in 


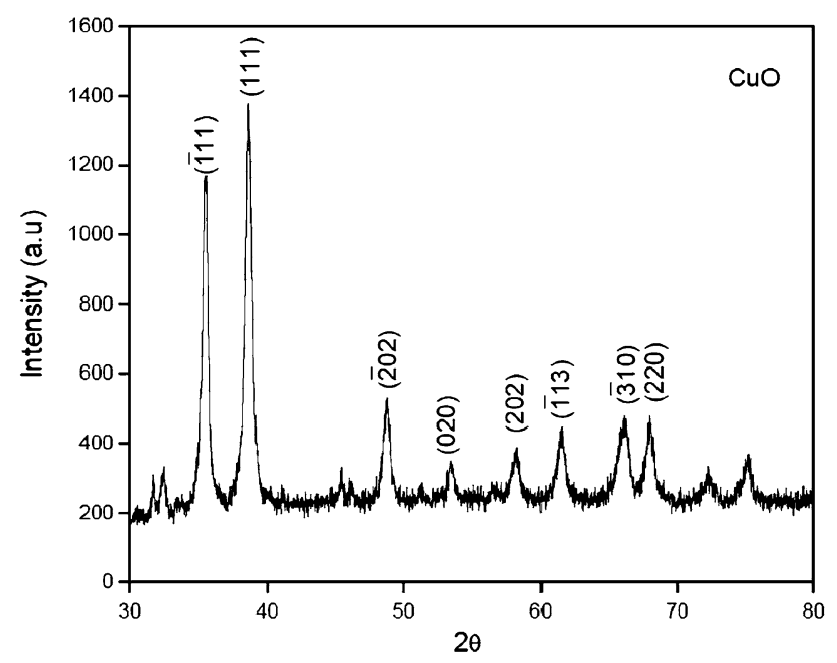

Fig. 1 X-ray diffractogram for the copper oxide nanoparticles

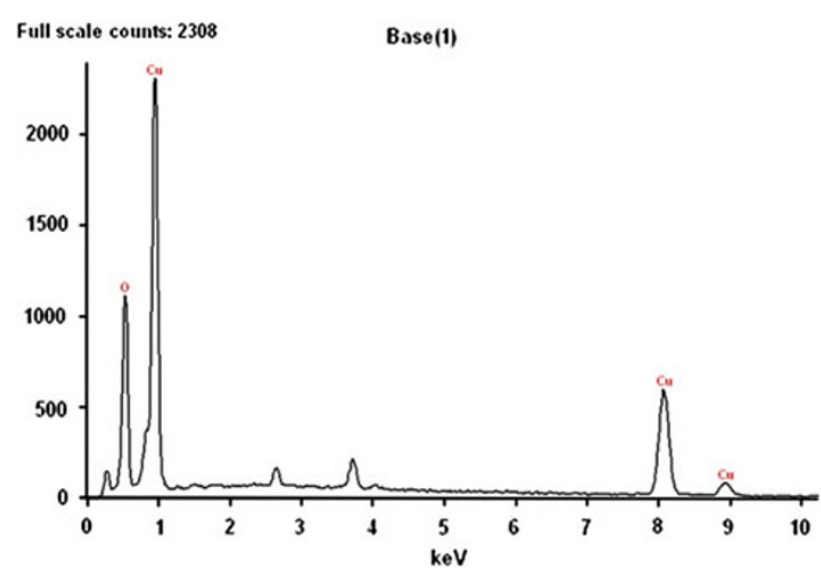

Fig. 2 EDXA image for the copper oxide nanoparticle

Fig. 2. Also the elemental analysis of the sample shows that the prepared sample is copper oxide which is in good agreement with the results of XRD.

\section{SEM}

Scanning electron microscope images were obtained for both copper oxide nanoparticle and copper oxide nanofluid by placing few drops of the dispersion on a copper grid and evaporating them prior to observation. Figure 3 shows the SEM image of Cuo nanoparticle which indicates that the morphology of particle is spherical in shape and shows very tiny agglomerates, and Fig. 4 shows the SEM image of $\mathrm{CuO}$ nanofluid, respectively.

Thermo gravimetric analysis

The thermo gravimetric analysis was carried out to confirm the thermal stability of the nanoparticle. Figure 5 shows the

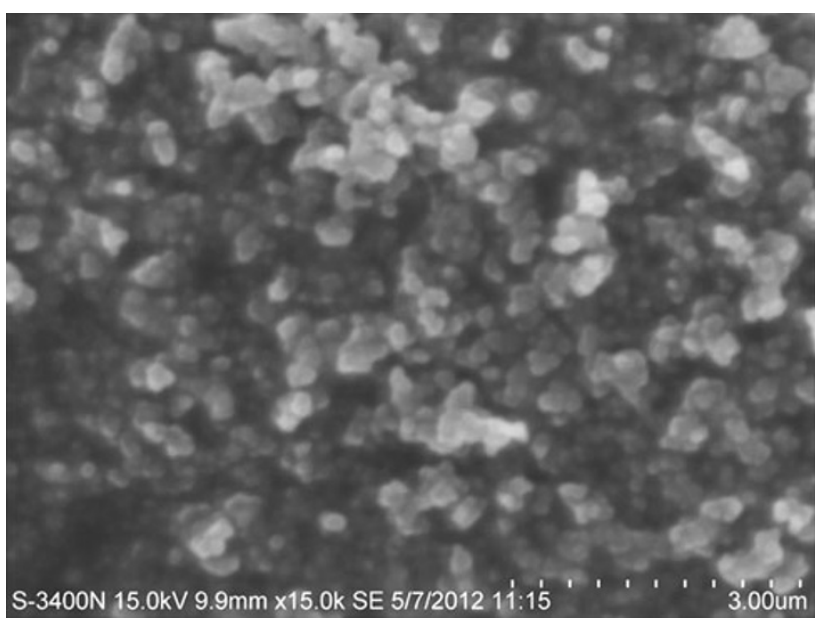

Fig. 3 SEM image of copper oxide nanoparticle

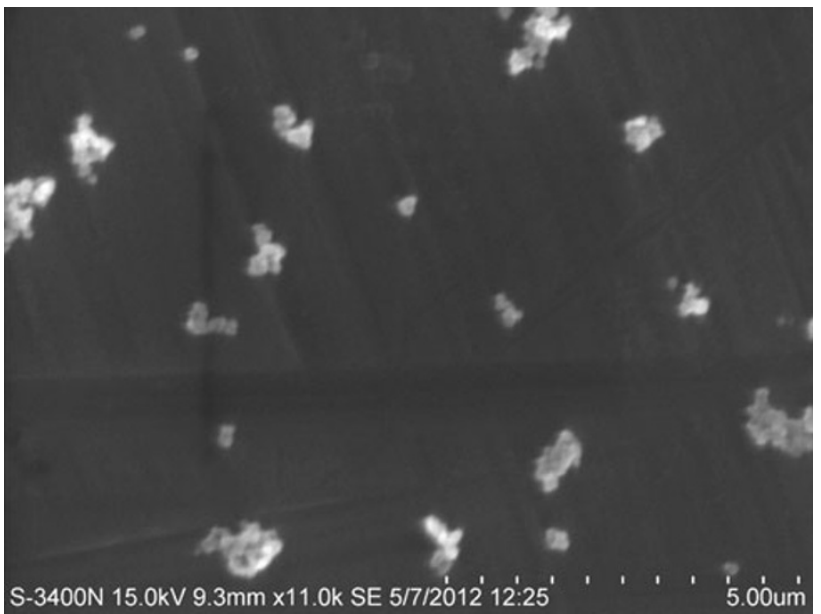

Fig. 4 SEM image of copper oxide nanofluid

thermo gravimetric analysis image of the copper oxide sample. The temperature was heated from 25 to $200{ }^{\circ} \mathrm{C}$ with a heating rate of $10^{\circ} \mathrm{C} / \mathrm{min}$. A decrease in weight occurs from 25 to $85.2{ }^{\circ} \mathrm{C}$ due to the vaporization of the residual water content, with weight loss of about $3.17 \%$. No significant loss is observed in the range of $85.2-200{ }^{\circ} \mathrm{C}$ where the weight remains constant indicating no oxidation behavior of the copper oxide nanoparticle. Darezereshki and Bakhtiari (2011) reported that the weight loss is $9.9 \mathrm{wt} \%$ in the temperature range of $100-400{ }^{\circ} \mathrm{C}$ for copper oxide nanoparticles which is $67.9 \%$ higher than the present weight loss.

Thermal conductivity

The thermal conductivity of $\mathrm{CuO}$ nanofluid was measured using a KD2 Pro thermal property analyser (Decagon Devices, Inc., USA). The KD2 Pro is a battery-operated, 


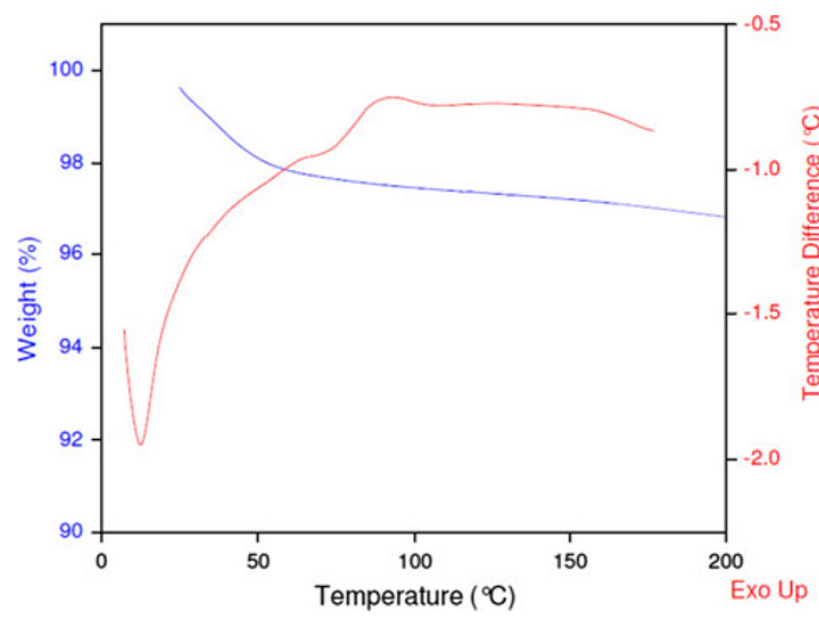

Fig. 5 Thermo gravimetric analysis of copper oxide nanoparticle

menu-driven device. It consists of a handheld microcontroller and sensor needles. The KD2's sensor needle contains both a heating element and a thermistor. The sensor needle can be used for measuring thermal conductivity of fluids in the range of $0.2-2 \mathrm{~W} / \mathrm{mK}$ with an accuracy of $\pm 5 \%$. The controller computes the thermal conductivity using the change in temperature $(\Delta \mathrm{T})$ - time data from

$K=\frac{q\left(\ln t_{2}-\ln t_{1}\right)}{4 \pi\left(\Delta T_{2}-\Delta T_{1}\right)}$

where $q$ is constant heat rate applied to an infinitely long and small line source, $\Delta T_{1}$ and $\Delta T_{2}$ are the changes in the temperature at times $t_{1}$ and $t_{2}$, respectively (Suresh et al. 2011)

The calibration of the sensor needle was carried out first by measuring thermal conductivity of distilled water. The measured values for distilled water is $0.611 \mathrm{~W} / \mathrm{mK}$ which is in agreement with the literature values of $0.613 \mathrm{~W} / \mathrm{mK}$ within $\pm 5 \%$ accuracy (Hagen 1999; Vargaftik 1975; Kothandaraman 2007). Thus, the measured thermal conductivity of copper oxide nanofluid is $0.698 \mathrm{~W} / \mathrm{mK}$ which showed an increase of $12.4 \%$ when compared to deionised water. Also, the values are higher when compared with Jwo et al. (2007) where he indicated about only $9.6 \%$ increase in thermal conductivity.

\section{Conclusion}

Thus, the copper oxide nanofluid has been synthesized using wet chemical method using copper chloride as a precursor, and the average size of the nanoparticle was found to be $20 \mathrm{~nm}$ which is calculated using De-Scherrer formula. Also, the thermal conductivity is measured using KD2 Pro thermal property analyser which indicates that there is a significant increase in thermal conductivity of the prepared copper oxide nanofluids compared to deionised water and the increase was found to be $12.4 \%$. Hence from the study, it can be concluded that the copper oxide nanofluid can be used for heat transfer applications due to their enhancement in thermal conductivity.

Open Access This article is distributed under the terms of the Creative Commons Attribution License which permits any use, distribution, and reproduction in any medium, provided the original author(s) and the source are credited.

\section{References}

Bennici S, Gervasini A, Ragaini V (2003) Preparation of highly dispersed $\mathrm{CuO}$ catalysts on oxide supports for de-NOx reactions. Ultrason Sonochem 10:61-64

Carnes CL, Klabunde KJ (2003) The catalytic methanol synthesis over nanoparticle metal oxide catalysts. J Mol Catal A: Chem 194:227-236

Choi SUS (1995) Enhancing thermal conductivity of fluids with nanoparticles. In: Signer DA, Wang HP (eds) Developments applications of non-newtonian flows, FED-vol. 231/MD-vol. 66, ASME, New York, pp 99-105

Darezereshki E, Bakhtiari F (2011) A novel technique to synthesis of tenorite $(\mathrm{CuO})$ nanoparticles from low concentration $\mathrm{CuSO}_{4}$ solution. J Min Metall Sect B-Metall 47:73-78

Eastman JA, Choi SUS, Li S, Yu W, Thompson LJ (2001) Anomalously increased effective thermal conductivities of ethylene glycol-based nanofluids containing copper nanoparticles. Appl Phys Lett 78:718

Frietsch M, Zudock F, Goschnick J, Bruns M (2000) CuO catalytic membrane as selectivity trimmer for metal oxide gas sensors. Sens Actuators B: Chem 65:379-381

Godson L, Raja B, Mohan Lal D, Wongwises S (2010) Enhancement of heat transfer using nanofluids-an overview. Renew Sustain Energy Rev 14:629-641

Hagen KD (1999) Heat Transfer with Applications. Prentice-Hall, Upper Saddle River

Jwo CS, Teng TP, Chang J (2007) A simple model to estimate thermal conductivity of fluid with acicular nanoparticles. J Alloys Compd 434-435:569-571

Kothandaraman CP, Subramanyan S (2007) Heat and mass transfer data book, 6th edn. New Age International, New Delhi

Kurihara LK, Chow GM, Schoen PE (1995) Nanocrystalline metallic powders and films produced by the polyol method. Nanostruct Mater 6:607-613

Lee S, Choi SUS, Li S, Eastman JA et al (1999) Measuring thermal conductivity of fluids containing oxide nanoparticles. Trans Am Soc Mech Eng 121:280-289

Li CH, Peterson GP (2006) Experimental investigation of temperature and volume fraction variations on the effective thermal conductivity of nanoparticles suspensions (nanofluids). J Appl Phys 99:084314

Liu MS, Lin MCC, Huang IT, Wang CC (2006) Enhancement of thermal conductivity with $\mathrm{CuO}$ for nanofluids. Chem Eng Technol 29:72-77

Murshed SMS, Leong KC, Yang C (2005) Enhanced thermal conductivity of $\mathrm{TiO}_{2}$-water based nanofluids. Int J Therm Sci 44:367-373

Sukhorukov YP, Loshkareva NN, Samokhvalov AA, Naumov SV, Moskvin AS, Ovchinnikov AS (1998) Magnetic phase transitions in optical spectrum of magnetic semiconductor $\mathrm{CuO}$. J Magn Magn Mater 183:356-358 
Suresh S, Venkitaraj KP, Selvakumar P, Chandrasekar M (2011) Synthesis of $\mathrm{Al}_{2} \mathrm{O}_{3}-\mathrm{Cu}$ /water hybrid nanofluids using two step method and its thermo physical properties. Colloids Surf A Physicochem Eng Asp 388:41-48

Vargaftik NB (1975) Tables of the thermophysical properties of liquids and gases, 2nd edn. Wiley, New York

Wang X, Xu X, Choi SUS (1999) Thermal conductivity of nanoparticle-fluid mixture. Thermophys Heat Transf 13:474-480

Xuan Y, Li Q (2003) Investigation on convective heat transfer and flow features of nanofluids. Int J Heat Transf 125:151-155
You TY, Niwa O, Tomita M, Andoo H, Suzuki M, Hirono S (2002) Characterization and electrochemical properties of highly dispersed copper oxide/hydroxide nanoparticles in graphite like carbon films prepared by RF sputtering method. Electrochem Commun 4:468-471

Zhu HT, Lin YS, Yin YS (2004) A novel one-step chemical method of preparation of copper nanofluids. J Coll Interface Sci 277: $100-103$ 